\title{
Harnessing the Power of Mobile Phone Technology to Improve Maternal Healthcare in Tanzania: Proposed Model
}

\author{
Ally S. Nyamawe \\ The University of Dodoma \\ College of Informatics and Virtual Education,
}

\author{
Hassan Seif \\ The University of Dodoma \\ College of Informatics and Virtual Education,
}

\begin{abstract}
In the effort to reduce maternal mortality rate, Millennium Development Goal (MDG) 5 was declared globally. Tanzania has no exception on this. Maternal mortality and morbidity is still an enduring challenge in Tanzania. In 2010 the Maternal Mortality Rate (MMR) was recorded at 454/100,000 live births. Despite of the devoted efforts and significant progress that Tanzania has made in reducing MMR, still the situation demands for more improved intervention. One among the contributors of maternal mortality is lack of education to expectant mothers. In this paper, a model with a pivotal role to extend health information accessibility to expectant mothers is proposed. A proposed model is exploiting the potential and power of mobile phones to deliver its services. Existing extended network coverage and expansion of mobile phone usage are the unprecedented advantages that would also be exploited.
\end{abstract}

\section{General Terms}

ICT for Health

\section{Keywords}

ICT, Maternal, Mortality Rate, Maternal Health Care, MDG.

\section{INTRODUCTION}

Most of the maternal mortalities and morbidities are mainly attributed by the known and avoidable causes. Lack of skilled attendants during delivery, lack of accessible health facilities and lack of education are named to be among the accelerating factors to maternal mortalities. Majority of Tanzanians are living in rural areas which are mainly marginalized. Rural areas are characterized by deficient of quality medical services, including accessible and equipped health centers, qualified medical practitioners and assured medical supplies. Limited of antenatal care services, nutritional education and postnatal care services increase the likelihood of adverse effects to maternal health and infants.

In recent years, maternal mortalities have received a special attention globally. The situation led to declaration of the Millennium Development Goal (MDG) 5 which is aiming at cutting down Maternal Mortality Ratio (MMR). Despite of encouraging improvements in reducing MMR in Tanzania, still more efforts need to be devoted to realize the dream of MDG 5. Blank A. et al. [1] contend that, in 2010, a tragic 287,000 maternal deaths are expected to have taken place world-wide. The largest burden of the reported maternal deaths, approximately $56 \%$ were carried by the countries in Sub-Saharan Africa corresponding to an average Maternal Mortality Ratio (MMR) of 500 deaths per 100,000 live births and a life time risk of 1 in 39 for a woman to die of maternal causes[2].
Furthermore, researches show that; one woman dies per minute in a child birth around the globe. Almost half of these deaths occur in Sub-Saharan Africa. Despite the effort made so far by many countries, but majority of these pregnant women fail to get full access of accurate information when they are pregnant [3].

Hunt P. et al. [4] contend that, if all women had access to the interventions for preventing or treating pregnancy and birth complications, an estimated 74 per cent of maternal deaths could have been avoided. To considerably cut down MMR, quality maternal health care services delivery is inexorable. Furthermore, [4] suggested that, numerous obstacles to the well-being of maternal will be removed if women will have access to technical services and information that can often prevent maternal mortality and morbidity.

To extend the accessibility of health information to expectant mothers, a model for a mobile based information system is presented in this paper. The rapid escalation of cellular network coverage and expansion of mobile phone usage has open up a new way of deploying services. Mobile phone technology coverage in Tanzania, has been growing tremendously [5]. In recent years, Mobile telephony technology has become powerful transformational tool and demonstrated a potential impact in health services delivery all over the global[6]. Tiwari P. [7], contends that, PC based systems are potentially taken over by mobile technology which support mobility needs of patients and medical practitioners. Furthermore, [7] contends that, with these advantages it is increasingly becoming possible to create innovative solutions for developing countries in delivering services and resolve problems facing the healthcare systems.

\section{RELATED WORKS}

Currently, there is a noticeable increase in number of mobile phones subscribers in developing countries, Tanzania included. The ubiquity of mobile technology offers tremendous opportunities for the healthcare industry to address one of the most dominant challenges: making healthcare more accessible, faster, better and cheaper [8]. In align to that, The Government of Tanzania through its " National Development Vision 2025" has recognize the importance of ICT in fulfilling this vision by developing and deploying a nationwide e-health system that supports medical facilities in the under-served areas. Not only that but also to promote the use of ICT to enhance efficiency, effectiveness and sustainability in the provision of services and basic utilities by supporting the development and deployment of nationwide e-health [9]. Hence there is a need for innovative technical solutions in health systems which can help to reduce MMR in Tanzania. Currently various countries have been implementing mobile applications which can be used to allow maternal to get various useful information before delivering. 
In Ghana they have implemented Mobile Technology for Community Health (MOTECH) which is a partnership between Ghana Health Service, Grameen Foundation and Columbia University's Mailman School of Public Health. The project aims to determine how to use mobile phones to increase the quantity and quality of prenatal and neonatal care in rural Ghana, with a goal of improving health outcomes for mothers and their newborns [10]

ChildCount+ is a community health events reporting and alerts platform aimed at empowering communities to improve child survival and maternal health. Using any standard phone, community health extension workers (CHEWs) are able to use text messages to register patients and send health reports to a central web dashboard that allows a health team to closely monitor the health of their community and reduce gaps in treatment. The location of this project was Tanzania, Kenya and Ghana but it is actively used in Kenya [11]

In Zambia and Ghana they implement a project named "Mwana" which focuses on using mobile technology to strengthen health services for mothers and infants in rural health care clinics. It was initially deployed in Zambia to deliver HIV test results instantly via SMS to rural clinics and reduce the delay between testing and treatment from several months to a few weeks [11].

Furthermore; TulaSalud's project was implemented in Guatemala with a vision of using ICT and mobile technology to reduce maternal and infant mortality and to monitor disease outbreaks in the remote highlands of Alta Verapaz, Guatemala, which has the largest rural and poor indigenous population in the region with limited access to health care services. Using mobile phone, TulaSaludi has been able to improve the flow of information between health professionals based in hospitals and community health workers (CHWs) in remote villages [11].

In Tanzania there are a number of projects which have been implemented to support the health of pregnant woman. MAMA (Mobile Alliance for Maternal Action) which is Healthy Pregnancy, Healthy Baby - Text Messaging Service offers Tanzanians free text messages in Swahili for pregnant women, mothers with newborns up to 16 weeks old, as well as supporters of pregnant women and new mothers (partners, friends and relatives) [12]. This project was challenged in utilizing a mobile health service, by the fact that, end-users had to live in an area, or ability to travel to an area, with network coverage, also in the case of text message-based services, they must be literate [12].

Wazazi Nipendeni (Parents Love Me) is a national Healthy Pregnancy and Safe Motherhood multi-media campaign in
Tanzania. The aim of the campaign is to harness mobile phone technology and text messaging to reduce maternal and infant mortality numbers by three-quarters by 2015 . It promotes its free text messaging (SMS) service by listing the short-code on its campaign materials. This messages provide information about antenatal care, family planning, malaria prevention, nutrition (for mother and baby), danger signs, individual birth plan, postpartum care and entertaining information such as fetal development milestones [13].

In Rwanda they have designed and implemented an innovative SMS-based alert system (RapidSMS-MCH) [14]. The system enables effective and real-time two-way communication for action between Community Health Workers (CHWs) at community level, and the rest of the health system through mobile phones. The expectation of the system is an improved access to antenatal care, postnatal care, institutional delivery and emergency obstetric care. Furthermore, the system provides a database for keeping clinical records of maternal care delivery [14].

\section{PROPOSED MODEL}

\subsection{Motivation}

In recent years, mobile phones have emerged to be the most hand held devices which support higher degree of mobility. A number of solutions are now deployed to be accessed via mobile phones to take advantage of their extended coverage that exists today. Taking that into consideration, the proposed model is employing the use of mobile phones in delivering health information to maternal in the effort to reduce MMR. In Tanzania, MMR is lessening at a very low speed and therefore more efforts are needed to cut it down significantly. Educating expectant mothers during prenatal and postnatal period is vital for their health and wellbeing of their infants.

\subsection{Overview of the proposed model}

A model is focusing toward delivering an innovative, low cost and user friendly solution for delivering health information to mothers during pregnancy and postnatal periods. Maternal, Traditional Birth Attendants (TBAs), Community Health Workers (CHWs) and Medical Practitioners at regional and referral hospitals are interlinked. This ensures comprehensive, timely and precise health services delivery to maternal. Pregnant women need to register to the system in order to receive appropriate health information (example, Nutritional issues), reminders (example, Antenatal Care Visiting) and warnings in case of acute indications. On top of that, a model ensures efficient data storage and processing and producing useful reports to facilitate decision making. 


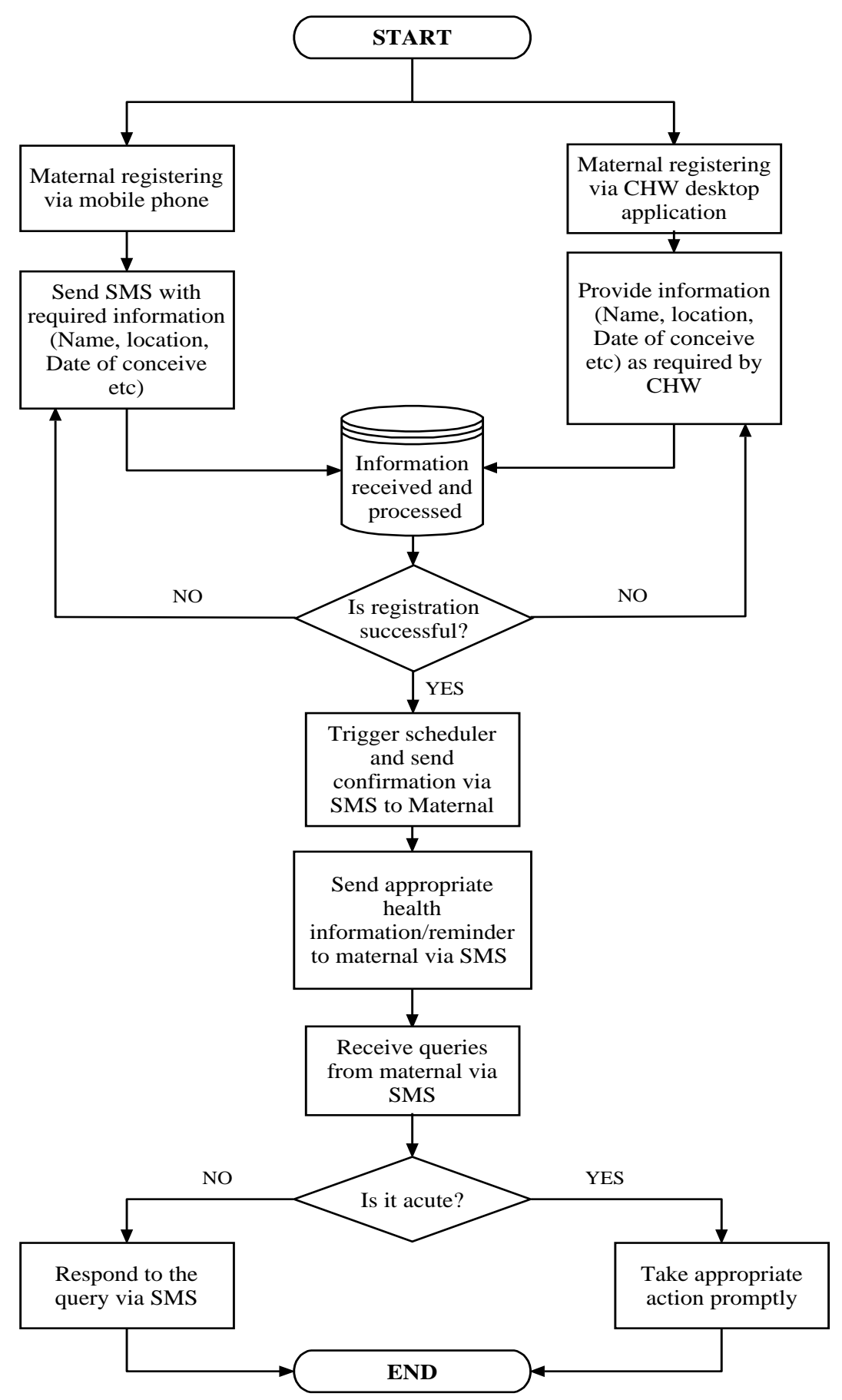

Fig. 1: A flowchart depicting the basic functioning of the proposed model

\subsection{Architecture of the proposed model}

To clearly demonstrate the basic functioning of the proposed model, we have summarized the flow of events in a flowchart as depicted in Figure 1.

The proposed model is made to be composed of two key layers namely; Application layer and Backend layer as depicted in Figure 2.

\subsubsection{Application Layer}

Application layer is consisting of four components which are:

\subsubsection{Traditional Birth Attendants (TBAs)}

As TBAs belongs to the same community with the pregnant women, they are vital in offering a quick assistance to mothers in case of an emergency and while looking to reach a nearest health facility. Therefore, there is a need to connect mothers to the closest TBAs. After official registration of TBA which is done by the CHW, TBA communicates with the rest of the system via SMS. To understand which TBA to contact in case of help, the system tracks and locates each and every TBA. Along with SMS notification, TBAs can be called for quick response.

\subsubsection{Medical Professional (MP)}

Medical Professional needs to be stationed with smartphone which are considered more powerful than traditional phone. Smartphone is needed simply because MP interacts with the backend layer through SMS and internet connectivity. 
Moreover, MP's smartphone is installed with an application which connects to the backend layer to retrieve information, receive queries from maternal and upload information to the server. The system automatically assigned a closest registered medical professional to maternal. The received queries from maternal will be channeled to a specific MP for prompt response. On a go, MP can download details or medical history of the maternal from the server to facilitate decision making.

\subsubsection{Maternal}

A pregnant woman accesses the system via SMS to perform registration, call for a help, retrieve reminders, ask questions and receive health information.

Registration via SMS requires less information. Pregnant woman needs to provide full name, location and date of conceive and sends the SMS to the server for registration. However, pregnant woman will be advised to visit the closest health facility to provide more details and track other medical records. After successful registration, a scheduler at the backend is automatically triggered to create a schedule for notifications and reminders. A registered pregnant woman is automatically assigned to the nearest available TBA, MP, Health Facility and CHW. Pregnant woman can send SMS to the server to call for a help, the assigned MP, TBA and CHW will be promptly notified for each to take appropriate action.

Pregnant woman receives as per schedule the reminders to visit antenatal clinic for checkups and medications. Reminder SMSs are well contextualized and in Swahili language to enhance more comprehension. In addition to that, a pregnant woman may ask questions regarding her health and in case she experiences any misbehaving in her body. The assigned MP is responsible to respond to the queries and prescribes accordingly.

Constantly, the registered pregnant woman receives health information from the server. Medical advises are given such as nutritional feeding, body exercises and food hygiene. Along with notification of expected date of delivery, expectant mother is also informed on how to prepare herself right before delivery and after delivery for the betterment of her health and newborn.

\subsubsection{Community Health Worker (CHW)}

CHW is a health attendant at the health facility nearest to pregnant woman. Pregnant woman visits health facility for antenatal clinics, medications and delivery. CHW connects to the server via mobile phone and workstation. Mobile phone allows for mobility as when the CHW is out of duty station, still can receive calls and notifications via SMS. To support CHW to register pregnant woman, update details (example, medical history), delete details, and retrieve reports, CHW's workstation accesses a web based application from the server. Workstation connects to the server via internet through a GSM Modem. Furthermore, CHW is privileged to manually alter notifications and reminders scheduling. In addition to that, CHW is responsible to register TBAs with their details and locations.

\subsubsection{Backend Layer}

At the backend of the application layer is where a processing zone (Backend layer) resides. The backend layer of the proposed model is composed of three key components; GSM modem, SMS gateway and Database server.

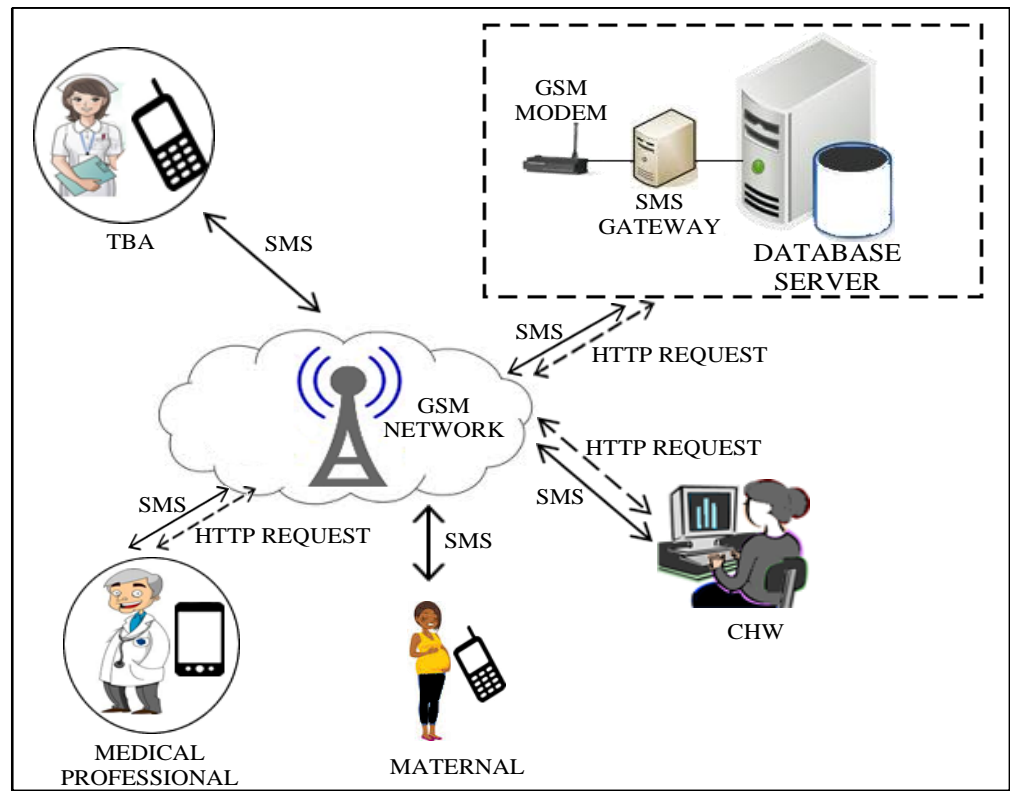

Fig. 2: Architecture of the proposed model

\subsubsection{GSM Modem}

A GSM modem is for receiving SMSs from application layer and sending back SMSs to the application layer. GSM Modem can also be used to provide internet connectivity.

\subsubsection{SMS Gateway}

To bridge GSM Modem and Database server, SMS Gateway is used. SMS Gateway provides services that are continuously running at background to listen for incoming messages and react accordingly. GSM Modem passes a message through to
SMS Gateway where it is going to be transformed to string of texts ready for manipulation. The manipulated texts are then sent to the underlying application stored in a database server for computation.

\subsubsection{Database Server}

The database Server is responsible for storage, computation and processing. The database Server hosts a web based application and the underlying database. A web based application receives queries from client applications 
(Maternal, MP and CHW) and act upon. A scheduler is part of the web based application; a scheduler is solely for scheduling reminders and notifications. Table 1 depicts simplified selected algorithms that are used by the web based application.

\section{CONCLUSION}

Tanzania is struggling towards achieving MDGs including MDG 5 which aims at reducing maternal mortality rate by 2015. Hence the efforts are needed in order to achieve these MDGs. The cheapness of mobile phone and penetration of mobile phone technology all over the country; suggest the need of integrating health sectors with mobile phone technology as among the solutions towards achieving MDG 5. By considering the proposed model, a mobile phone can improve maternal healthcare through simplified health enquiries, calling for a help and retrieving of reminders. In addition to that, expectant mothers will be able to receive more health information relating to pregnancy, medications during pregnancy, expected date of delivery, diet, development of pregnancy and so on. In short, a pregnant woman gets well informed during prenatal and postnatal periods. We would therefore suggest that, there is a need of implementing projects based on the proposed model to improve maternal healthcare in Tanzania.

\section{Table 1: Simplified algorithm}

**Receive query from Maternal

If (a query is for registration) Then

1. Register maternal

2. Trigger Scheduler for reminders

Else If (a query is for enquiry) Then

1. Retrieve the details of the assigned MP

2. Send the enquiry to the assigned MP

Else

Something is wrong, notify accordingly

End If

**

\section{** Send reminders}

- Retrieve current system date

- Check the database for all appointments scheduled for the next two days.

- Retrieve the details of the corresponding maternal

- Compose a reminder

- Send a reminder

$* *$

**Receive response from $M P$

- Check Query ID for which the response is made

- Retrieve details of the maternal posted the query

- Check MP response

If (the issue is acute) Then

- Retrieve information of the assigned TBA and CHW

- $\quad$ Notify assigned TBA and CHW for immediate action

Else (Non acute) Then

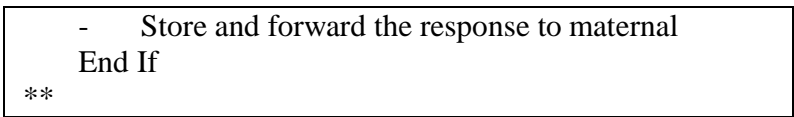

5. REFERENCE

[1] A. Blank, H. Prytherch, J. Kaltschmidt, A. Krings, F. Sukums, N. Mensah, A. Zakane, S. Loukanova, L. L. Gustafsson, R. Sauerborn, and W. E. Haefeli, "'Quality of prenatal and maternal care : bridging the know-do gap ' ( QUALMAT study ): an electronic clinical decision support system for rural Sub-Saharan Africa," BMC Med. Inform. Decis. Mak., 2013

[2] WHO, UNICEF, UNFPA, and WORLD BANK, "Trends in Maternal Mortality: 1990- 2010,” 2012.

[3] Africa progress panel policy, "Maternal Health :Investing in the Lifeline of Healthy Societies and Economies," 2010.

[4] H. Paul and B. Judith, "Reducing Maternal Mortality. The contribution of the right to the highest attainable standard of health."

[5] A. K. Hassan and D. Semkwiji, "The Role of Mobile Phones on Sustainable Livelihood," 2011.

[6] C. Z. Qiang, M. Yamamichi, V. Hausman, and D. Altman, "Mobile Applications for the Health Sector,", December, 2011.

[7] P. Tiwari, "Providing healthcare services in rural India : Innovative application of mobile technology," Heal. Care Informatics Rev. Online, vol. 14, no. 2, pp. 3-9, 2010.

[8] PWC, "Emerging mHealth: Paths for growth," 2012.

[9] $\mathrm{MOH}$, "e Health in Tanzania National Strategic Plan Final Draft," 2010.

[10] Gramen Foundation, "Mobile technology for community health in Ghana," 2011.

[11] J. Lemaire, "Scaling up mobile health elements necessary for the mhealth in developing countries," 2011.

[12] MAMA (Mobile alliance for maternal action), "healthy pregnancy , healthy baby text messaging service tanzania," 2013.

[13] CDC Foundation, "mHealth Text Messaging Campaign in Tanzania.".

[14] F. Ngabo, J. Nguimfack, F. Nwaigwe, C. Mugeni, D. Muhoza, D. R. Wilson, J. Kalach, R. Gakuba, C. Karema, and A. Binagwaho, "Designing and Implementing an Innovative SMS-based alert system (RapidSMS-MCH) to monitor pregnancy and reduce maternal and child deaths in Rwanda," Pan Afr. Med. J., 2012. 